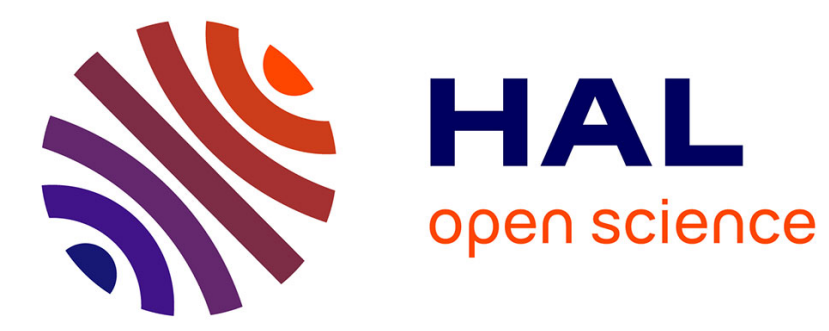

\title{
Underwater topography invisible for surface waves at given frequencies
}

\author{
Anne-Sophie Bonnet-Ben Dhia, Sergei Nazarov, Jari Taskinen
}

\section{To cite this version:}

Anne-Sophie Bonnet-Ben Dhia, Sergei Nazarov, Jari Taskinen. Underwater topography invisible for surface waves at given frequencies. Wave Motion, 2015, 57, 10.1016/j.wavemoti.2015.03.008 . hal01114632

\section{HAL Id: hal-01114632 \\ https://hal.inria.fr/hal-01114632}

Submitted on 9 Feb 2015

HAL is a multi-disciplinary open access archive for the deposit and dissemination of scientific research documents, whether they are published or not. The documents may come from teaching and research institutions in France or abroad, or from public or private research centers.
L'archive ouverte pluridisciplinaire HAL, est destinée au dépôt et à la diffusion de documents scientifiques de niveau recherche, publiés ou non, émanant des établissements d'enseignement et de recherche français ou étrangers, des laboratoires publics ou privés. 


\title{
Underwater topography invisible for surface waves at given frequencies
}

\author{
Anne-Sophie Bonnet-Ben Dhia, Sergey A. Nazarov and Jari Taskinen
}

February 9, 2015

\begin{abstract}
We consider scattering of surface waves modelled by the linear water wave equation in an unbounded two-dimensional domain of finite depth, at a given frequency and a given incidence. Using asymptotic analysis for small perturbations of the bottom shape, we build a fixed-point equation whose unique solution is a shape which cannot be detected by a distant observer. The method works at any incidence except $\pi / 4$.
\end{abstract}

Keywords: linear water waves, asymptotic analysis, scattering problem, invisibility, cloaking, fixed point theorem

\section{Introduction.}

Related to the current progress in realizing artificial metamaterials, a great interest is devoted to different ways for achieving the cloaking of an object, making it invisible for electromagnetic waves [1]. Of course, the same question can be investigated for other types of waves, acoustic waves or water waves for instance. This has been already proved to work experimentally $[2,3]$. If perfect invisibility, at all frequencies and for all incident waves, remains an unreachable dream, some nice results can be obtained by considering only waves in a given frequency range. Going further, in the context of waveguides, one can take benefit of the presence at a given frequency of only a finite number of propagating waves. In other words, for a receiver located far from the perturbation, the echoes due to this later are resumed in a finite number of complex numbers, the so-called scattering coefficients. The question then reduces to proving the existence of perturbations canceling these coefficients. This remark has been exploited successfully in [4] for two-dimensional acoustic waveguides. However, it has been noticed that the method was rapidly deteriorating when increasing the frequency, related to the fact that the number of propagating modes is an increasing function of the frequency. This partially motivated the present study, where water waves are considered. Indeed, contrary to acoustic waveguides, there exist only one guided wave whatever the frequency is. Some other differences between the cases of acoustics and water waves will be discussed later. 
More precisely, we consider a water layer of finite depth $d$, and we aim to find geometric distortions of the bottom which are not detectable, at a given frequency, by an observer located far from the distortion. Assuming that the perturbation of the bottom is invariant in the horizontal direction $x$, the scattering by an incident surface wave reduces to a two-dimensional problem set in the $(y, z)$ cross-section of the fluid domain, with the frequency $\omega$ and the $k_{x}$ component of the wave vector as parameters. We consider that the cloaking is obtained if the scattered field, due to the perturbation of the bottom, is composed of evanescent modes, so that it decreases exponentially with the distance to the perturbation. Equivalently, it means that the reflexion coefficient $\mathbf{r}$ and the transmission coefficient $\mathbf{t}$ (which are a priori complex numbers) are such that $\mathbf{r}=0$ and $\mathbf{t}=1$. A possible approach to find a perturbation of the bottom satisfying such conditions is to use a numerical algorithm of optimization. This has been done in [5] where the object to be cloaked is a vertical three-dimensional cylinder and in [6] where submerged steps and horizontal plates are considered in two dimensions. Our contribution here is quite different. On one hand, our results are weaker since we only get the invisibility for small perturbations of the bottom. But this is counterbalanced by the two following nice properties:

1. First, we obtain a theoretical proof of existence of invisible perturbations, which is not the case in [5] or [6]. At the same time, we prove the convergence of the fixed-point algorithm to this invisible profile.

2. Secondly, our method allows to some extent to design the main features of the perturbation, which is then only slightly modulated by the algorithm in order to achieve the perfect cloaking.

Our technique for the construction of the invisible bottom profiles $h$ is inspired by the technique used to prove the enforced stability of trapped modes (or embedded eigenvalues) in $[7,8]$. More precisely, the method aims to build, for any given small $\varepsilon$, a perturbation of the bottom whose amplitude is of order $\varepsilon$ and which is completely invisible, in the sense that the reflection $\mathbf{r}$ and the distortion of the transmission $\mathbf{t}-1$ satisfy $\mathbf{r}=\mathbf{t}-1=0$. To do that, we search the profile of the bottom perturbation $h$ as a linear combination of a small number of given functions $H_{j}$. These functions have to fulfill some orthogonality and normalization conditions, such that the coefficients of the linear combination solve a fixed-point equation, which can be proved to be a contraction under appropriate conditions. More details will be given later, but let us mention that one can build many different invisible profiles, by changing either the value of $\varepsilon$ (smaller than some limiting value) or by changing the functions $H_{j}$. We will show that the method works for all frequencies and all angles of incidence, except the angle $\pi / 4$, where the differential of $\mathbf{r}$ with respect to $h$ is vanishing. Explaining this exception and getting rid of this condition is an open question. A similar difficulty occurred in the case of acoustic waveguides, where it was not possible to achieve $\mathbf{t}=1$ but only the weaker condition $|\mathbf{t}|=1$.

A limitation of the approach is that it allows to build only small invisible perturbations of the bottom, since the contraction property is lost for large values 
of $\varepsilon$. A new idea is investigated in the present paper, to provide larger invisible perturbations. It consists of applying the previous approach, replacing the initial straight bottom by some invisible profile. Namely, one may try to apply this result repeatedly to "cultivate" an invisible profile of large amplitude. The method becomes less explicit and we are not able to prove that the degeneracy of the differential of the functional $h \longmapsto(\mathbf{r}, 1-\mathbf{t})$ can be avoided. However, all the requirements are quite computable, and the perturbation analysis can help to develop numerical algorithms for producing invisible perturbations of larger magnitude.

The outline of the paper is the following. In Section 2, we present the method to build invisible perturbations of a straight bottom of small amplitudes. The next step where the invisible profile itself is perturbed while preserving invisibility is described in Section 3. The theoretical justifications of the asymptotic analysis used in Section 2 and 3 are given in Section 4. Some possible extensions are finally discussed in the last section. For example we explain how to create an invisible perturbation of the bottom for a prescribed finite set of frequencies $\omega_{1}, \ldots, \omega_{N}$ or $x$-wave numbers $k_{1}, \ldots, k_{J}$.

\section{Invisible perturbations of the bottom of small amplitude.}

We denote by $\Pi$ the two-dimensional strip $\mathbb{R} \times(-d, 0) \ni(y, z)$, which describes the cross-section of the water domain with constant depth $d$. Then, for a given profile function $h$, we denote by $\Pi^{h}$ the cross-section of the perturbed water domain, defined as follows (see Figure 1):

$$
\Pi^{h}=\{(y, z) ; y \in \mathbb{R}, 0>z>-d-h(y)\} .
$$

The bottom perturbation is assumed to be invariant in $x$, smooth and situated in the region $\{|y|<L\}$ for some $L>0$, so that $h \in C_{c}^{\infty}(-L, L)$, which is the space of infinitely smooth functions with compact support in the segment $(-L, L)$. The main problem is formulated as finding profile functions $h$ such that after passing over the obstacle, the surface wave of a given frequency and a given incidence produces only an exponentially decreasing scattered field.

\subsection{Plane waves of the unperturbed domain.}

Let us present the mathematical model for surface waves used in this paper. In the unperturbed three dimensional water layer $\mathbb{R} \times \Pi$, the propagation of

water waves is described by the velocity potential $\Phi$ satisfying the equation of incompressibility

$$
\Delta \Phi=0, \quad(x, y, z) \in \mathbb{R} \times \Pi,
$$

with the kinematic Steklov condition

$$
\partial_{z} \Phi=g^{-1} \omega^{2} \Phi, \quad(x, y) \in \mathbb{R}^{2}, z=0,
$$




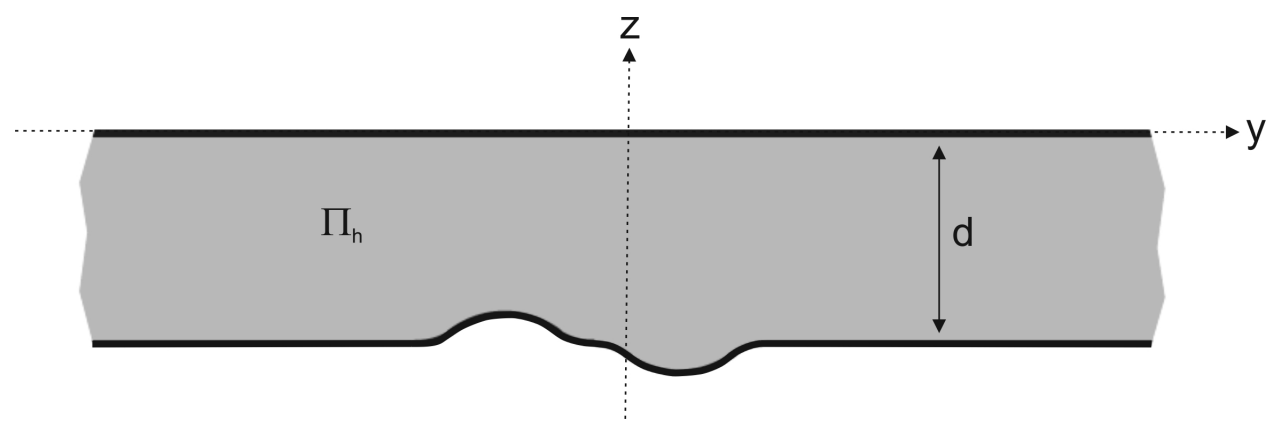

Figure 1: Bottom topography.

on the free surface, with the acceleration of gravity $g>0$ and the frequency of time harmonic oscillations $\omega>0$, and the Neumann non-penetration condition

$$
\partial_{z} \Phi=0, \quad(x, y) \in \mathbb{R}^{2}, z=-d,
$$

on the bottom. Particular solutions are the plane waves of the form

$$
\Phi(x, y, z)=w(z) e^{i(k x+\ell y)}
$$

with

$$
w(z)=\cosh (\gamma(z+d))
$$

where $\gamma$ is the unique solution of the dispersion relation

$$
\gamma \tanh (\gamma d)=g^{-1} \omega^{2}
$$

and the components $k$ and $\ell$ of the wave vector are such that

$$
\gamma^{2}=k^{2}+\ell^{2}
$$

\subsection{Definition of invisible bottom perturbations.}

Now we consider a perturbed water domain defined by (1). The perturbation of the bottom causes scattering of a plane wave (5). Since the perturbation profile $h$ is assumed constant in the $x$-direction, the scattering problem remains two-dimensional (cf. Section 5.3 for a more general case). For a given frequency $\omega$, the angle of incidence of the plane wave with respect to the perturbation is fixed by the parameter $k$, which has to be chosen such that $k<\gamma$. The value $k=0$ corresponds to the case of normal incidence, cf. Figure 2. Then the total velocity potential for the scattering problem takes the form:

$$
\Phi(x, y, z)=\varphi(y, z) e^{i k x}
$$




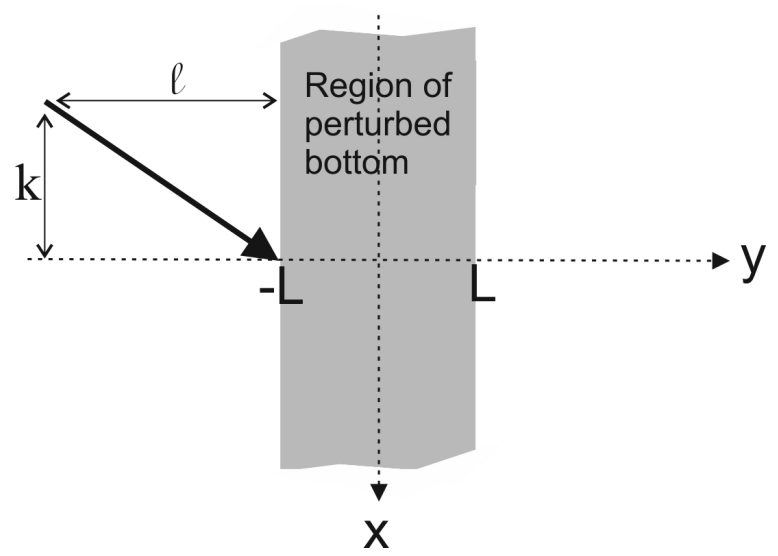

Figure 2: The direction of the incident wave.

where $\varphi$ has to satisfy the following equations

$$
\begin{aligned}
-\partial_{y}^{2} \varphi(y, z)-\partial_{z}^{2} \varphi(y, z)+k^{2} \varphi(y, z) & =0, \quad(y, z) \in \Pi^{h}, \\
\partial_{z} \varphi(y, 0) & =g^{-1} \omega^{2} \varphi(y, 0), y \in \mathbb{R}, \\
\partial_{n} \varphi(y,-d-h(y)) & =0, \quad y \in \mathbb{R} .
\end{aligned}
$$

In the last equation, $\partial_{n}$ stands for the derivative along the outward normal. We introduce the normalized waves

$$
w_{ \pm}(y, z)=\frac{e^{ \pm i \ell y} w(z)}{\|w\|_{L^{2}(-d, 0)}}
$$

where $w$ is given by (6). Because of the wavenumber $\pm \ell$, the wave $w_{ \pm}$travels in the channel $\Pi$ from $\mp \infty$ to $\pm \infty$. In general, the wave $w_{+}$, incoming from the left side of the channel scatters from the bottom perturbation and thus gives rise to a reflection to the left and a transmission to the right. This can be summarized by writing the solution of the homogeneous problem (9)-(11) in the form

$$
\varphi(y, z)=\chi_{-}(y)\left(w_{+}(y, z)+\mathbf{r} w_{-}(y, z)\right)+\mathbf{t} \chi_{+}(y) w_{+}(y, z)+\widetilde{\varphi}(y, z),
$$

where $\chi_{ \pm}$are smooth cut-off functions such that

$$
\chi_{ \pm}(y)=1 \text { for } \pm y>2 L \text { and } \chi_{ \pm}(y)=0 \text { for } \pm y<L,
$$

$\mathbf{t}$ and $\mathbf{r}$ are the complex transmission and reflection coefficients, respectively, and the remainder $\widetilde{\varphi}$ decays exponentially as $|y| \rightarrow \infty$. The perturbation of the profile is said to be invisible if and only if

$$
\mathbf{r}=0 \text { and } \mathbf{t}=1
$$


One can easily prove the energy conservation which gives the following relation between the scattering coefficients

$$
|\mathbf{t}|^{2}+|\mathbf{r}|^{2}=1
$$

so that the invisibility is achieved as soon as

$$
\mathbf{r}=0 \text { and } \Im m(\mathbf{t})=0 .
$$

We also mention that since $\overline{w_{+}}=w_{-}$, one can easily check that if the perturbation is invisible for the incident wave $w_{+}$incoming from the left, it is also invisible for the incident wave $w_{-}$incoming from the right.

\subsection{Asymptotic analysis.}

Now we consider a water domain $\Pi^{h}$ with a small perturbation of the straight bottom, taking the function

$$
h:=\varepsilon H \in C_{c}^{\infty}(-L, L)
$$

for the bottom topography in (1), where $\varepsilon>0$ is a small parameter. Our first objective is to derive the first terms of the asymptotic expansion of $\varphi$ with respect to $\varepsilon$.

We accept the simplest asymptotic ansatz for regularly perturbed domains,

$$
\varphi(y, z)=w_{+}(y, z)+\varepsilon \varphi^{\prime}(y, z)+\ldots,
$$

cf. [9, Ch. 5]. Notice that both $w_{+}$and $\varphi^{\prime}$ are originally defined in the straight strip $\Pi$ but can be extended smoothly to the lower half-plane; in this way the expansion (19) is well-understood in $\Pi^{h}$, too. The dots in (19) stand for higher order terms inessential for our asymptotic analysis. Inserting (19) into (9)-(10), we readily conclude that

$$
\begin{array}{r}
-\partial_{y}^{2} \varphi^{\prime}(y, z)-\partial_{z}^{2} \varphi^{\prime}(y, z)+k^{2} \varphi^{\prime}(y, z)=0 \quad, \quad(y, z) \in \Pi, \\
\partial_{z} \varphi^{\prime}(y, 0)=g^{-1} \omega^{2} \varphi^{\prime}(y, 0) \quad, \quad y \in \mathbb{R} .
\end{array}
$$

To derive a boundary condition on the rectified bottom, we use the Taylor formula and the representation

$\partial_{n}=\left(1+\varepsilon^{2}\left|\partial_{y} H(y)\right|^{2}\right)^{-1 / 2}\left(-\partial_{z}-\varepsilon \partial_{y} H(y) \partial_{y}\right)=-\partial_{z}-\varepsilon \partial_{y} H(y) \partial_{y}+\ldots$

for the normal derivative. We then have

$$
\begin{aligned}
0= & \partial_{n} \varphi(y,-d-\varepsilon H(y)) \\
= & -\partial_{z} w_{+}(y,-d-\varepsilon H(y))-\varepsilon \partial_{y} H(y) \partial_{y} w_{+}(y,-d-\varepsilon H(y))+\ldots \\
& -\varepsilon \partial_{z} \varphi^{\prime}(y,-d-\varepsilon H(y))+\ldots \\
= & -\partial_{z} w_{+}(y,-d)+\varepsilon H(y) \partial_{z}^{2} w_{+}(y,-d)-\varepsilon \partial_{y} H(y) \partial_{y} w_{+}(y,-d) \\
& -\varepsilon \partial_{z} \varphi^{\prime}(y,-d)+\ldots
\end{aligned}
$$


Finally, using the fact that $\partial_{z} w_{+}(y,-d)=0$ and

$$
-\partial_{y}^{2} w_{+}-\partial_{z}^{2} w_{+}+k^{2} w_{+}=0, \quad(y, z) \in \Pi
$$

we obtain

$$
-\partial_{z} \varphi^{\prime}(y,-d)=\partial_{y}\left(H(y) \partial_{y} w_{+}(y,-d)\right)-H(y) k^{2} w_{+}(y,-d) \quad, \quad y \in \mathbb{R} .
$$

We now consider the problem $(20,21,23)$ satisfied by $\varphi^{\prime}$ in the unperturbed domain $\Pi$. It is known (see, e.g., [10]) that the problem $(20,21,23)$ has a unique solution subject to the Sommerfeld radiation condition

$$
\varphi^{\prime}(y, z)=\mathbf{r}^{\prime} \chi_{-}(y) w_{-}(y, z)+\mathbf{t}^{\prime} \chi_{+}(y) w_{+}(y, z)+\widetilde{\varphi}^{\prime}(y, z),
$$

where the notation is quite similar to (13), in particular $\mathbf{r}^{\prime}$ and $\mathbf{t}^{\prime}$ are some complex coefficients and the remainder $\widetilde{\varphi}^{\prime}$ decays exponentially as $|y| \rightarrow \infty$. Then, comparing (13), (19) and (24), we obtain the following expansions for $\mathbf{r}$ and $\mathbf{t}$ :

$$
\mathbf{t}=1+\varepsilon \mathbf{t}^{\prime}+\varepsilon^{2} \tilde{\mathbf{t}}_{\varepsilon}, \mathbf{r}=\varepsilon \mathbf{r}^{\prime}+\varepsilon^{2} \tilde{\mathbf{r}}_{\varepsilon} .
$$

To compute the coefficients $\mathbf{r}^{\prime}$ and $\mathbf{t}^{\prime}$, we insert the function $\varphi^{\prime}$ and the waves $w_{\alpha}, \alpha= \pm$, into the Green formula on the long $(R \rightarrow+\infty)$ rectangle $(-R, R) \times$ $(-d, 0)$ and obtain

$$
\begin{aligned}
& \int_{-R}^{R} \overline{w_{\alpha}(y,-d)} \partial_{z} \varphi^{\prime}(y,-d) d y \\
= & \sum_{ \pm} \pm \int_{-d}^{0}\left(\overline{w_{\alpha}( \pm R, z)} \partial_{y} \varphi^{\prime}( \pm R, z)-\varphi^{\prime}( \pm R, z) \overline{\partial_{y} w_{\alpha}( \pm R, z)}\right) d z
\end{aligned}
$$

On one hand, it results from (24) that the right-hand side of (26) is equal to $i \mathbf{r}^{\prime}$ if $\alpha=-$ and $i \mathbf{t}^{\prime}$ if $\alpha=+$. On the other hand, using (23) and integrating by parts in the segment $(-L, L)$, we prove that the left-hand side of $(26)$ converts into

$$
\int_{-L}^{L} H(y)\left(\partial_{y} w_{+}(y,-d) \overline{\partial_{y} w_{\alpha}(y,-d)}+k^{2} w_{+}(y,-d) \overline{w_{\alpha}(y,-d)}\right) d y .
$$

Thus we finally obtain the expressions

$$
\begin{aligned}
\mathbf{t}^{\prime} & =-i \frac{k^{2}+\ell^{2}}{N} \int_{-L}^{L} H(y) d y, \\
\mathbf{r}^{\prime} & =-i \frac{k^{2}-\ell^{2}}{N} \int_{-L}^{L} e^{2 i \ell y} H(y) d y,
\end{aligned}
$$


where

$$
N=2 \ell\|w\|^{2}=d \ell\left(1+\frac{\sinh (2 \gamma d)}{2 \gamma d}\right) .
$$

Notice than for large $d, N$ behaves like $\ell e^{2 \gamma d} /(2 \gamma)$. This means that the scattering effect of a perturbation of amplitude $\varepsilon$ decreases exponentially with the depth of the water domain, which is not surprising since the velocity potential of water waves decreases exponentially with the depth.

Let us also mention that approximate formulas

$$
\mathbf{t} \sim 1+\varepsilon \mathbf{t}^{\prime}, \mathbf{r} \sim \varepsilon \mathbf{r}^{\prime}
$$

for the scattering coefficients are similar to the well-known Born approximation for the scattering by weak heterogeneities.

\subsection{The fixed-point equation}

Consider a bottom perturbation $h=\varepsilon H_{0}$ and suppose for instance that $H_{0}$ is such that

$$
\int_{-L}^{L} H_{0}(y) d y=\int_{-L}^{L} e^{2 i \ell y} H_{0}(y) d y=0 .
$$

From (25) and (27), we deduce that the scattering effects of the perturbation are of order $\varepsilon^{2}$. It is already interesting but not sufficient since our objective is to get the perfect invisibility, ensured by (17). The idea is then to look for a bottom perturbation $h=\varepsilon H$ with $H$ of the form

$$
H(y)=H_{0}(y)+\sum_{j=1}^{3} \tau_{j} H_{j}(y),
$$

where the functions $H_{j} \in C_{c}^{\infty}(-L, L), j=0,1,2,3$ satisfy the following conditions

$$
\begin{aligned}
\int_{-L}^{L} H_{j}(y) d y & =\delta_{j, 1}, \\
\int_{-L}^{L} \cos (2 \ell y) H_{j}(y) d y & =\delta_{j, 2}, \\
\int_{-L}^{L} \sin (2 \ell y) H_{j}(y) d y & =\delta_{j, 3},
\end{aligned}
$$

$\delta_{j, p}$ denoting the Kronecker symbol. The idea is that the coefficients $\left(\tau_{1}, \tau_{2}, \tau_{3}\right)$ will be tuned in order to satisfy (38). We expect them to be of order $\varepsilon$ so that 
they can compensate the scattering effects of order $\varepsilon^{2}$ due to $H_{0}$. In the sequel, we set $\tau=\left(\tau_{1}, \tau_{2}, \tau_{3}\right)$.

Going further, using (25), (27) and (28)-(31), we obtain

$$
\begin{array}{r}
\mathbf{t}(\varepsilon, \tau)=1-\varepsilon i \frac{k^{2}+\ell^{2}}{N} \tau_{1}+\varepsilon^{2} \tilde{\mathbf{t}}(\varepsilon, \tau), \\
\mathbf{r}(\varepsilon, \tau)=0-\varepsilon i \frac{k^{2}-\ell^{2}}{N}\left(\tau_{2}+i \tau_{3}\right)+\varepsilon^{2} \tilde{\mathbf{r}}(\varepsilon, \tau) .
\end{array}
$$

In Section 4 we will demonstrate that the functions

$$
(\varepsilon, \tau) \mapsto \mathbf{t}(\varepsilon, \tau) \text { and }(\varepsilon, \tau) \mapsto \mathbf{r}(\varepsilon, \tau)
$$

are analytic in the cylinder

$$
\mathcal{Q}=\left\{(\varepsilon, \tau) \in \mathbb{R}^{4} ;|\varepsilon| \leq \varepsilon_{0},|\tau| \leq \tau_{0}\right\}
$$

for suitable positive $\varepsilon_{0}$ and $\tau_{0}$. Moreover, the estimates

$$
|\tilde{\mathbf{t}}(\varepsilon, \tau)| \leq C_{0} \text { and }|\tilde{\mathbf{r}}(\varepsilon, \tau)| \leq C_{0},
$$

which justify the asymptotic analysis, are also verified there, see (69).

As a result, the relations

$$
\Im m(\mathbf{t}(\varepsilon, \tau))=0, \Im m(\mathbf{r}(\varepsilon, \tau))=0, \Re e(\mathbf{r}(\varepsilon, \tau))=0
$$

are converted into the system of transcendental equations

$$
\begin{aligned}
\left(k^{2}+\ell^{2}\right) \tau_{1} & =\varepsilon N \Im m(\tilde{\mathbf{t}}(\varepsilon, \tau)) \\
\left(k^{2}-\ell^{2}\right) \tau_{2} & =\varepsilon N \Im m(\tilde{\mathbf{r}}(\varepsilon, \tau)) \\
\left(k^{2}-\ell^{2}\right) \tau_{3} & =-\varepsilon N \Re e(\tilde{\mathbf{r}}(\varepsilon, \tau)) .
\end{aligned}
$$

We assume that

$$
k \neq \ell
$$

and dividing by the numbers on the left hand sides, rewrite the system (38) in the vector form

$$
\tau=T^{\varepsilon}(\tau)
$$

where

$$
T^{\varepsilon}(\tau)=\varepsilon N\left(\begin{array}{c}
\Im m(\tilde{\mathbf{t}}(\varepsilon, \tau)) \\
\Im m(\tilde{\mathbf{r}}(\varepsilon, \tau)) \\
\Re e(\tilde{\mathbf{r}}(\varepsilon, \tau))
\end{array}\right)
$$

Due to factor $\varepsilon$, the estimate (36) and the analyticity of the functions (34) the operator $T^{\varepsilon}: \mathbb{R}^{3} \rightarrow \mathbb{R}^{3}$ becomes a contraction in the ball

$$
\mathcal{B}=\left\{\tau \in \mathbb{R}^{3} ;\left|\tau-T^{\varepsilon}(0)\right| \leq \varrho_{0}\right\},
$$

if $\varrho_{0}$ and $\varepsilon$ are small enough. Hence, the contraction mapping principle implies the existence of a unique solution $\tau=\tau(\varepsilon) \in \mathcal{B}$ of (40) such that the estimate

$$
|\tau(\varepsilon)| \leq C_{0} \varepsilon
$$

holds in addition.

The desired invisible profile function (28) has been found. 


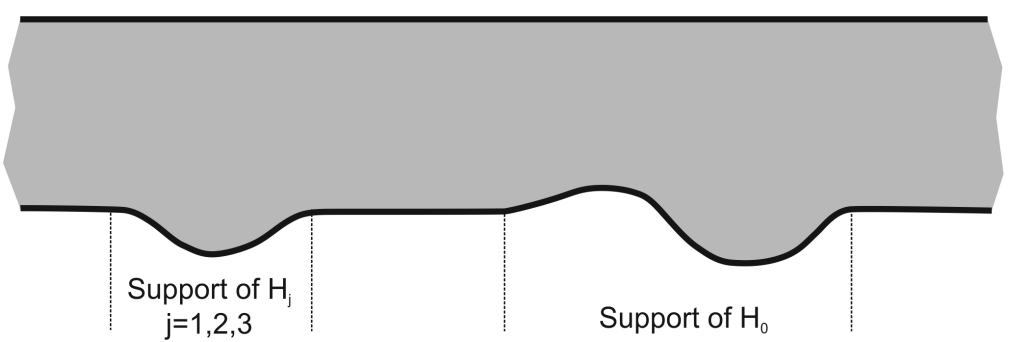

Figure 3: The case of distant tuning.

\subsection{Discussing the result.}

Clearly, all the requirements (29)-(31) can easily be satisfied. The inequality (42) shows that

$$
H(y)=H_{0}(y)+O(\varepsilon),
$$

and therefore the profile $h(y)=\varepsilon H(y)$ is mainly defined by $H_{0}$ while the functions $\tau_{j}(\varepsilon) H_{j}$ play the role of small correction terms which besides depend on the parameter $\varepsilon$. Let us emphasize that there are infinitely many different choices for $H_{0}$, so that we can build infinitely many completely different invisible perturbations of the bottom.

Notice that we do not pose any condition on the supports of $H_{j}, j=1,2,3$. In particular they can be situated at some distance of the support of $H_{0}$ as in Figure 3, so that a distant tuning is also possible.

The condition (29) implies that

$$
V(\varepsilon)=\int_{-L}^{+L} h(y) d y=\varepsilon \int_{-L}^{+L} H(y) d y=\varepsilon \tau_{1} .
$$

This means that the volume increment of the bottom perturbation becomes $O\left(\varepsilon^{2}\right)$, which may be disappointing for some types of applications. Also condition (29) forces $H_{0}$ (and then $H$ ) to be sign-changing.

A way to allow profile functions of constant sign is to weaken the objective, imposing only $\mathbf{r}=0$. Then $|\mathbf{t}|=1$ by the energy conservation but $\mathbf{t}$ can be different from 1 , which means that the perturbation may produce a phase shift of the wave.

To get such a non-reflecting perturbation profile $h$ (such that $\mathbf{r}=0$ ), it suffices to consider a bottom perturbation $h=\varepsilon H$ with $H$ of the form

$$
H(y)=H_{0}(y)+\sum_{j=2}^{3} \tau_{j} H_{j}(y)
$$

where the functions $H_{j} \in C_{c}^{\infty}(-L, L), j=0,2,3$ satisfy the conditions (30) and (31) only (condition (29) is no more necessary). 


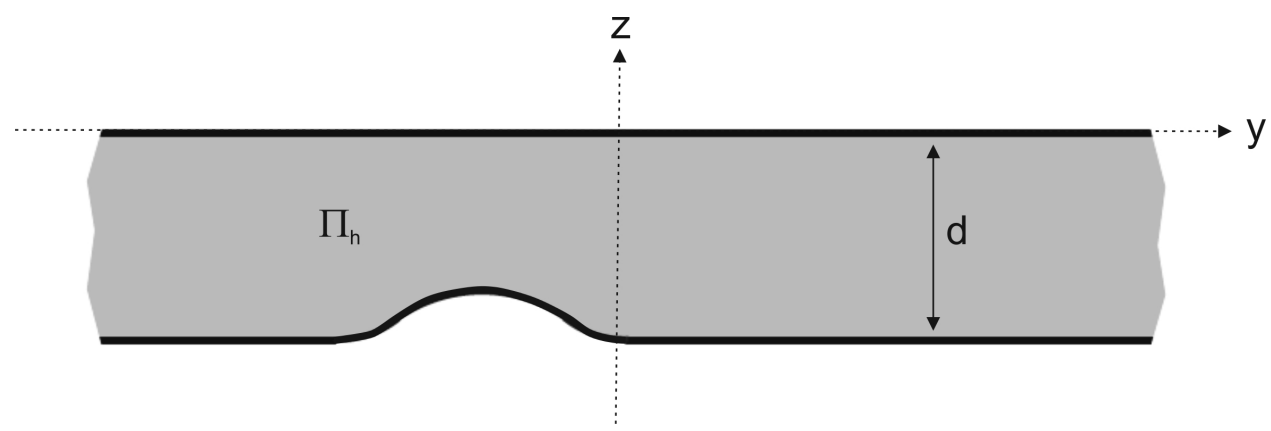

Figure 4: A bottom perturbation of constant sign.

Notice in particular that (30) and (31) may be satisfied by a non-positive or by a non-negative function (cf. Figure 4 ) with

$$
V_{0}=-\int_{-L}^{L} H_{0}(y) d y \neq 0 .
$$

Then the total volume increment of the bottom perturbation becomes $V(\varepsilon)=$ $\varepsilon V_{0}+O\left(\varepsilon^{2}\right)$ and thus stays not zero for small $\varepsilon$.

The condition (39) means that the angle of incidence has to be different from $\pi / 4$. It was introduced because, if $k=\ell$, the left hand sides of the last two equations in (38) vanish, and this makes our previous conclusion on the existence of the vector $\tau(\varepsilon)$ impossible. The authors do not know a physical reason for this restriction. Notice that for $k=\ell$, the reflexion coefficient $\mathbf{r}$ is always of order $\varepsilon^{2}$, but we cannot prove at the moment that it can vanish for some perturbation.

Let us finally mention that, as the product $\varepsilon N$ appears in (38), we expect that we will have to take a smaller $\varepsilon$ to ensure the contraction property, when $N$ increases. In particular, the method should deteriorate very rapidly (exponentially) when the depth $d$ of the water domain increases. It should also deteriorate when the incidence becomes grazing $(\ell \rightarrow 0)$ or tends to $\pi / 4$. Indeed the division by $\ell$ or $\left(k^{2}-\ell^{2}\right)$ will provide large values of $T^{\varepsilon}$ when $\ell$ or $k-\ell$ respectively is small.

\section{Perturbation analysis of invisible profiles.}

This section contains a generalization of the approach and methods of Section 2. As a starting point we assume to be given a suitable "invisible" profile $h$, and we aim to present a method which allows to make a new perturbation of $h$ while still preserving the invisibility properties. As a consequence, this procedure can be iterated to yield more general invisible bottom profiles, hopefully with larger perturbations. 


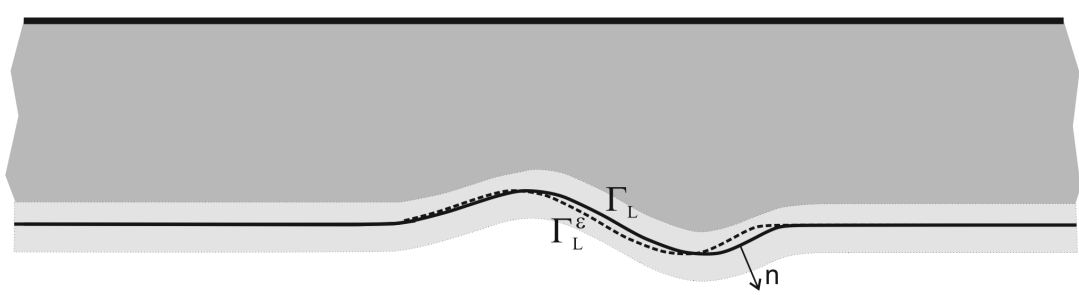

Figure 5: Tubular neighbourhood $\mathcal{V}$.

\subsection{Assumptions on the reference profile.}

We assume to be given a reference profile $h$ in (1), such that the solution $\varphi$ of the problem (9)-(11) with the form (13) satisfies the invisibility conditions (17), i.e., the propagating wave (5) is not affected at infinity by this particular shape. In the following, it will be convenient to denote this potential $\varphi$ by $\varphi^{h}$.

We assume that there is no trapped mode at the frequency $\omega$ for this particular underwater topography. This means that any solution of the homogeneous problem (9)-(11) which belongs to $L^{2}\left(\Pi^{h}\right)$, or equivalently decays at infinity, is nothing but null.

All these assumptions are met for example by the invisible profiles studied in Section 2, but $h$ does not necessarily need to be of that form.

The bottom perturbation is described as follows. Let $\mathcal{V}$ like in Figure 5 be a tubular neighborhood of the curve $\Gamma_{L}=\{(y, z):|y|<L, z=-d-h(y)\}$ endowed with the natural curvilinear coordinate system $(s, n)$, where $n$ is the oriented distance to $\Gamma_{L}, n \geq 0$ outside $\Pi^{h}$ and $s \in\left(0, s_{L}\right)$ is the arc length measured along $\Gamma_{L}$ from the point $(-L,-d)$. Let $F \in C_{c}^{\infty}\left(0, s_{L}\right)$ and $\varepsilon$ again a small positive parameter. We define the perturbed curve

$$
\Gamma_{L}^{\varepsilon}=\left\{(y, z) \in \mathcal{V}: s \in\left(0, s_{L}\right), n=\varepsilon F(s)\right\},
$$

and the water domain $\Pi^{h, \varepsilon F}$ which lies between the free surface $\mathbb{R} \times\{0\}$ and the perturbed bottom $\Gamma^{\varepsilon}$ consisting of the arc (44) and the two semi-axes $(-\infty,-L]$ and $[L,+\infty)$. Our assumptions ensure that $F$ vanishes near the points $( \pm L,-d)$ so that $\Gamma^{\varepsilon}$ is still a smooth curve. We will next derive sufficient conditions for $F$, which ensure that the invisibility properties are preserved. The procedure for the construction of the asymptotics remains similar to that in Section 2, but concomitant calculations become a bit more complicated.

\section{$3.2 \quad$ Asymptotic analysis.}

Given $h$ and $\varphi^{h}$ as explained in Section 3.1, we introduce the ansatz generalizing (24):

$$
\varphi(y, z)=\varphi^{h}(y, z)+\varepsilon \varphi^{\prime}(y, z)+\varepsilon^{2} \widetilde{\varphi}(y, z),
$$


So, in (45), $\varphi$ and $\varphi^{h}$ stand for velocity potentials in $\Pi^{h, \varepsilon F}$ and $\Pi^{h}$, respectively, generated by the same incoming wave $w_{+}(y, z)$ on the left sides of these channels. The functions $\varphi$ and $\varphi^{\prime}$ are defined in $\Pi^{h}$, but if necessary, they can be extended smoothly to the neighborhood of $\Gamma_{L}$ covering the set $\Pi^{h, \varepsilon F} \backslash \Pi^{h}$.

Clearly, $\varphi^{\prime}$ satisfies the Helmholtz equation (9) and the Steklov condition (10). Let us derive the boundary condition satisfied by $\varphi^{\prime}$ on the bottom $\Gamma$ of $\Pi^{h}$. We keep the notation $\varphi^{\prime}(s, n)$ also in the curvilinear coordinates. Since

$$
\nabla=\left(\partial_{n},(1+\kappa(s) n)^{-1} \partial_{s}\right),
$$

where $\kappa$ is the curvature of $\Gamma$, the normal derivative $\partial_{n^{\varepsilon}}$ on $\Gamma^{\varepsilon}$ looks as follows:

$$
\begin{aligned}
& \frac{\partial}{\partial n^{\varepsilon}}=\left(1+\left|\frac{\varepsilon^{2}\left|\partial_{s} F(s)\right|^{2}}{(1+\varepsilon \kappa(s) F(s))^{4}}\right|\right)^{-1 / 2}\left(\frac{\partial}{\partial n}-\frac{\varepsilon \partial_{s} F(s)}{(1+\varepsilon \kappa(s) F(s))^{2}} \frac{\partial}{\partial s}\right) \\
= & \partial_{n}-\varepsilon \partial_{s} F(s) \partial_{s}+\ldots
\end{aligned}
$$

Hence, similarly to (27) we obtain

$$
\begin{aligned}
& \partial_{n^{\varepsilon}} \varphi(s, \varepsilon F(s)) \\
= & \partial_{n} \varphi^{h}(s, \varepsilon F(s))-\varepsilon \partial_{s} F(s) \partial_{s} \varphi^{h}(s, \varepsilon F(s))+\varepsilon \partial_{n} \varphi^{\prime}(s, \varepsilon F(s))+\ldots \\
= & \partial_{n} \varphi^{h}(s, 0)+\varepsilon F(s) \partial_{n}^{2} \varphi^{h}(s, 0)-\varepsilon \partial_{s} F(s) \partial_{s} \varphi^{h}(s, 0)+\varepsilon \partial_{n} \varphi^{\prime}(s, 0)+. .
\end{aligned}
$$

We now recall the Neumann boundary condition $\partial_{n} \varphi^{h}(s, 0)=0$ and the formula

$$
(1+\kappa(s) n)^{-1} \partial_{n}\left((1+\kappa(s) n) \partial_{n}\right)+(1+\kappa(s) n)^{-1} \partial_{s}\left((1+\kappa(s) n)^{-1} \partial_{s}\right)
$$

for the Laplacian in the curvilinear coordinates. Thus, $\partial_{n}^{2} \varphi^{h}(s, 0)=-\partial_{s}^{2} \varphi^{h}(s, 0)+$ $k^{2} \varphi^{h}(s, 0)$, and the boundary condition on $\Gamma$ reads as

$$
\partial_{n} \varphi^{\prime}(s, 0)=\partial_{s}\left(F(s) \partial_{s} \varphi^{h}(s, 0)\right)-k^{2} F(s) \varphi^{h}(s, 0) .
$$

As a consequence of the assumed non-existence of trapped modes, the problem (9), (10), (48) admits a unique solution $\varphi^{\prime}$ subject to the radiation conditions (24). To compute the arising coefficient $s_{+}^{\prime}$ we insert $\varphi^{\prime}$ and $\varphi$ into the Green formula on the rectangle $(-R, R) \times(-d, 0)$. Making use of (48) and integrating by parts along $\Gamma_{L}$ we deduce analogously to (26) that

$$
\begin{aligned}
& \int_{0}^{s_{L}} F(s)\left(\left|\partial_{s} \varphi^{h}(s, 0)\right|^{2}+k^{2}\left|\varphi^{h}(s, 0)\right|^{2}\right) d s \\
= & -\int_{0}^{s_{L}} \overline{\varphi^{h}(s, 0)} \partial_{n} \varphi^{\prime}(s, 0) d s \\
= & \lim _{R \rightarrow \infty} \sum_{ \pm} \pm \int_{-d}^{0}\left(\overline{\varphi^{h}( \pm R, z)} \partial_{y} \varphi^{\prime}( \pm R, z)-\varphi^{\prime}( \pm R, z) \overline{\partial_{y} \varphi^{h}( \pm R, z)}\right) d z \\
= & i \mathbf{t}^{\prime} .
\end{aligned}
$$


In the same way we get

$$
\int_{0}^{s_{L}} F(s)\left(\left(\partial_{s} \varphi^{h}(s, 0)\right)^{2}+k^{2}\left(\varphi^{h}(s, 0)\right)^{2}\right) d s=i \mathbf{r}^{\prime} .
$$

We emphasize that the integrand on the left in (49) involves moduli of the functions $\partial_{s} \varphi^{h}$ and $\varphi^{h}$, but in (50) the functions themselves. In particular the coefficient $\mathbf{t}^{\prime}$ is purely imaginary.

\subsection{The fixed-point equation.}

As in Section 2 we work with the representation

$$
F(s)=F_{0}(s)+\sum_{j=1}^{3} \tau_{j} F_{j}(s)
$$

involving the vector $\tau=\left(\tau_{1}, \tau_{2}, \tau_{3}\right)$ of small parameters and the functions $F_{q} \in$ $C_{c}^{\infty}\left(0, s_{L}\right)$. In view of $(49)$ and $(50)$, we define the three following functions:

$$
\begin{aligned}
G_{1}(s)= & \left|\partial_{s} \varphi^{h}(s, 0)\right|^{2}+k^{2}\left|\varphi^{h}(s, 0)\right|^{2}, \\
G_{2}(s)= & \left|\Re e\left(\partial_{s} \varphi^{h}(s, 0)\right)\right|^{2}-\left|\Im m\left(\partial_{s} \varphi^{h}(s, 0)\right)\right|^{2} \\
& +k^{2}\left(\left|\Re e\left(\varphi^{h}(s, 0)\right)\right|^{2}-\left|\Im m\left(\varphi^{h}(s, 0)\right)\right|^{2}\right), \\
G_{3}(s)= & 2 \Re e\left(\partial_{s} \varphi^{h}(s, 0)\right) \Im m\left(\partial_{s} \varphi^{h}(s, 0)\right)+2 k^{2} \Re e\left(\varphi^{h}(s, 0)\right) \Im m\left(\varphi^{h}(s, 0)\right) .
\end{aligned}
$$

We first impose to $F_{0}$ the following three orthogonality conditions

$$
\int_{0}^{s_{L}} F_{0}(s) G_{p}(s) d s=0, p=1,2,3 .
$$

Then we have to assume that the functions $G_{p}$ are linearly independent (this will be discussed in the next section), so that it is possible to find three functions $F_{j}$ such that

$$
\int_{0}^{s_{L}} F_{j}(s) G_{p}(s) d s=\delta_{p, j}, p, j=1,2,3 .
$$

Owing to (49), (50), (53) and (54), we obtain that:

$$
\Im m\left(\mathbf{t}^{\prime}(\varepsilon, \tau)\right)=-\tau_{1}, \Im m\left(\mathbf{r}^{\prime}(\tau)\right)=-\tau_{2}, \Re e\left(\mathbf{r}^{\prime}(\varepsilon, \tau)\right)=\tau_{3},
$$

and it is straightforward to convert the relations (17) into the abstract equation (40). The operator $T^{\varepsilon}$ in (40) remains a contraction in the ball (41) with some radius $\varrho_{0}>0$, because the functions (34) will be proven to be smooth in Section 4. Thus the contraction mapping principle again yields a unique solution $\tau=\tau(\varepsilon) \in \mathcal{B}$ and the estimate (41) for some constant $C_{0}$.

The desired perturbation profile (51) is now constructed by assuming (54) and (53). 


\subsection{Discussing the orthogonality and normalization con- ditions.}

Since $\varphi^{h}(s, 0)$ in (52) is not known explicitly, it is difficult to make conclusions about the linear independence of the functions $G_{1}, G_{2}$ and $G_{3}$, in (52). Moreover, we know that they can be dependent, because for the particular case $h=0$, one has $\Pi^{h}=\Pi$ and $\varphi^{h}=w_{+}$and therefore

$$
G_{2}(s)+i G_{3}(s)=\frac{k^{2}-\ell^{2}}{N} e^{2 i \ell y}
$$

which vanishes at $\ell=k$, that is for the incidence of $\pi / 4$; we have put the restriction (39) just to avoid null coefficients of $\tau_{2}$ and $\tau_{3}$ in (38) and to reduce the relations (37) to the solvable equation (40).

Evidently, neither $\Re e\left(\varphi^{h}(s, 0)\right)$ nor $\Im m\left(\varphi^{h}(s, 0)\right)$ vanish on any arc $\gamma \subset \Gamma_{L}$ of positive length (otherwise they would satisfy the Helmholtz equation (9) with both homogeneous Dirichlet and Neumann conditions and thus become null everywhere in $\left.\Pi^{h}\right)$. The same is true for the derivative $\partial_{s} \varphi^{h}(s, 0)$, cf. [11]. Hence, it is very easy to satisfy the first normalization condition (54) with $j=p=1$. The other two conditions $(j=p=2,3)$ need a different argument.

Suppose we consider an invisible profile $\varphi^{h}$ obtained by the method presented in section 2 . Then, by a continuity argument, it is clear that the linear independence of the functions $G_{1}, G_{2}$ and $G_{3}$ holds at least for $\ell \neq k$ and $\varepsilon$ small enough. Then an idea could be to use the iterative procedure explained in the beginning of this section, with a small $\varepsilon$ at each iteration. We can hope to obtain by this technique larger invisible perturbations than by increasing $\varepsilon$ in the initial approach, described in Section 2.

Finally, in the general case, there is no computational obstruction to verify, if $(54)$ can be met or not.

\section{Justification of asymptotics.}

To make the analysis in Sections 2 and 3 rigorous, it is necessary to treat the remainder terms in (19), (33), and (45). However, we only consider the last two of these in details, since the analysis performed in Section 2 can be regarded as a special case of Section 3.

\subsection{Operator formulation.}

We next present the suitable function spaces, as well as equations and radiation conditions satisfied by the remainder $\widetilde{\varphi}$. We solve the equations in Sections 4.2 and 4.3, and also show there that the solutions have the desired properties already used in Section 3.

The Kondratiev space (weighted Sobolev space) $W_{\beta}^{q}\left(\Pi^{h}\right)$ is the completion of the linear set $C_{c}^{\infty}\left(\Pi^{h}\right)$ of compactly supported infinitely smooth functions 
with respect to the norm

$$
\left\|\psi ; W_{\beta}^{q}\left(\Pi^{h}\right)\right\|=\sum_{p=0}^{q}\left\|e^{\beta|y|} \nabla^{p} \psi ; L^{2}\left(\Pi^{h}\right)\right\|,
$$

where $q=0,1, \ldots$ and $\beta \in \mathbb{R}$ are the smoothness and weight exponents and $\nabla^{p} \psi$ is the collection of all $p$ th order partial derivatives of $\psi$. In the case $\beta>0$ these functions decay exponentially at infinity. By $W_{\beta}^{1 / 2}\left(\partial \Pi^{h}\right)$ we understand the space of traces of functions in $W_{\beta}^{1}\left(\Pi^{h}\right)$ with the intrinsic norm

$$
\left\|\Psi ; W_{\beta}^{1 / 2}\left(\partial \Pi^{h}\right)\right\|=\inf \left\{\left\|\psi ; W_{\beta}^{1}\left(\Pi^{h}\right)\right\| ; W_{\beta}^{1}\left(\Pi^{h}\right) \ni \psi=\Psi \text { on } \partial \Pi^{h}\right\} .
$$

We associate to the inhomogeneous problem (9)-(11),

$$
\begin{aligned}
-\partial_{y}^{2} \psi(y, z) & -\partial_{z}^{2} \psi(y, z)+k^{2} \psi(y, z)=f(y, z), \quad(y, z) \in \Pi^{h}, \\
\partial_{z} \psi(y, 0) & -g^{-1} \omega^{2} \psi(y, 0)=f_{0}(y), \quad y \in \mathbb{R}, \\
-\partial_{n} \psi(y,-d-h(y)) & =f_{1}(y), \quad y \in \mathbb{R}
\end{aligned}
$$

the mapping

$$
A_{\beta}^{h}: W_{\beta}^{2}\left(\Pi^{h}\right) \rightarrow W_{\beta}^{0}\left(\Pi^{h}\right) \times W_{\beta}^{1 / 2}\left(\partial \Pi^{h}\right) \times W_{\beta}^{1 / 2}\left(\partial \Pi^{h}\right), \psi \mapsto\left(f, f_{0}, f_{1}\right)
$$

which is continuous for any $\beta \in \mathbb{R}$ but has "good" properties only under appropriate restrictions on the weight index (see [12] and e.g. [14, Ch. 3 and 5]).

To fix $\beta$, we note that the remainder $\widetilde{\varphi}$ in (13) satisfies the problem (57) with the right hand sides $f_{0}=f_{1}=0$ and $f \in C_{c}^{\infty}\left(\Pi^{h}\right)$,

$$
f(y, z)=\sum_{ \pm}\left(\partial_{y}^{2} \chi_{ \pm}(y)+2 \partial_{y} \chi_{ \pm}(y) \partial_{y}\right)\left(s_{ \pm} w_{ \pm}(y, z)+\delta_{ \pm,-} w_{+}(y, z)\right),
$$

where $s_{+}=\mathbf{t}, s_{-}=\mathbf{r}$ and we have the Kronecker delta of signs at the end. Applying the Fourier decomposition in the straight subdomains $\Pi_{ \pm}:=$ $\left\{(y, z) \in \Pi^{h} ; \pm y>2 \ell\right\}$, one shows that $\widetilde{\varphi}$ gains the decay rate $O\left(e^{-\beta_{0}|y|}\right)$, where $\beta_{0}=\sqrt{k^{2}+\mu_{1}^{2}}$ and $\mu_{1} d \in(\pi / 2, \pi)$ is the first positive root of the equation $\mu \tan (\mu d)=-g^{-1} \omega^{2}$. The constant $\beta_{0}$ is associated to the first evanescent mode in the channel. We now fix $\beta$ just by requiring

$$
\beta \in\left(0, \beta_{0}\right) .
$$

According to general results in [12] (see also [14, Ch. 2 and 5]), our assumption on the absence of trapped modes in (9)-(11) means that the operator (58) with the exponent (60) is a Fredholm monomorphism. We still use the weighted space with attached asymptotics, cf. [14, Ch.6] and [13], namely the space $\mathcal{W}_{\beta}^{2}\left(\Pi^{h}\right)$ which is composed of functions

$$
\psi(y, z)=\sum_{ \pm} \chi_{ \pm}(y) a_{ \pm} w_{ \pm}(y, z)+\widetilde{\psi}(y, z)
$$


where $a_{ \pm} \in \mathbb{C}$ and $\widetilde{\psi} \in W_{\beta}^{2}\left(\Pi^{h}\right)$, and is supplied with the norm

$$
\left\|\psi ; \mathcal{W}_{\beta}^{2}\left(\Pi^{h}\right)\right\|=\left(\left|a_{+}\right|^{2}+\left|a_{-}\right|^{2}+\left\|\widetilde{\psi} ; W_{\beta}^{2}\left(\Pi^{h}\right)\right\|^{2}\right)^{1 / 2} .
$$

Note that, similarly to (24), the expansion (61) is to be regarded as radiation conditions for the problem (57). Furthermore, being a proper skew-symmetric extension of the operator (58), the operator

$$
\mathcal{A}_{\beta}^{h}: \mathcal{W}_{\beta}^{2}\left(\Pi^{h}\right) \rightarrow W_{\beta}^{0}\left(\Pi^{h}\right) \times W_{\beta}^{1 / 2}\left(\partial \Pi^{h}\right) \times W_{\beta}^{1 / 2}\left(\partial \Pi^{h}\right)
$$

becomes an isomorphism, a useful fact in the later application of the perturbation theory of Banach space operators.

\subsection{Error estimates.}

We extend $\varphi^{h}$ and $\varphi^{\prime}$ smoothly to $\Pi^{h} \cup \mathcal{V} \supset \Pi^{h, \varepsilon F}$ (the neighborhood $\mathcal{V}$ was defined in Section 3.1) and observe that the difference (cf. (45))

$$
\varepsilon^{2} \widetilde{\varphi}=\varphi-\varphi^{h}-\varepsilon \varphi^{\prime}
$$

satisfies the problem (57) with $\Pi^{h, \varepsilon F}$ instead of $\Pi^{h}$, with the radiation conditions (61), and with the right hand sides written in the form

$$
f(y, z)=\varepsilon^{2} \widetilde{f}^{\varepsilon}(y, z), f_{0}(y)=0, f_{1}(y)=\varepsilon^{2} \widetilde{f}_{1}^{\varepsilon}(y),
$$

Here $\widetilde{f}^{\varepsilon}$ vanishes for $|y|>2 \ell$, since $\varphi^{h}$ and $\varphi^{\prime}$ both solve (9) in $\Pi^{h}$ and $\varphi$ in $\Pi^{h, \varepsilon F}$ (see the remarks after (45)); also $\widetilde{f}_{1}^{\varepsilon}$ is null for $|y|>2 \ell$ due to the boundary conditions satisfied by the terms in (64). Hence, $\widetilde{f}^{\varepsilon} \in W_{\beta}^{0}\left(\Pi^{h, \varepsilon F}\right)$ and $\tilde{f}_{1}^{\varepsilon} \in W_{\beta}^{1 / 2}\left(\partial \Pi^{h, \varepsilon F}\right)$, respectively, for any $\beta$, in particular for (60). Moreover, the norms of these functions are small: since $\varphi^{h}$ and $\varphi^{\prime}$ both obey the Helmholtz equation (9), their $C^{2+J}$-smooth extensions provide the $C^{J}$-extension of the expression $\left(\partial_{y}^{2}+\partial_{z}^{2}+k^{2}\right)\left(\varphi^{h}+\varepsilon \varphi^{\prime}\right)=0$ and, hence, the Taylor formula gives

$$
\left\|\tilde{f}^{\varepsilon} ; W_{\beta}^{0}\left(\Pi^{h, \varepsilon F}\right)\right\| \leq c \varepsilon^{-2} \varepsilon^{J} \varepsilon^{1 / 2},
$$

where $\varepsilon^{-2}$ comes from the left hand side of (64) and $\varepsilon^{1 / 2}=O\left(\operatorname{mes}_{2}\left(\Pi^{h, \varepsilon F} \backslash\right.\right.$ $\left.\Pi^{h}\right)^{1 / 2}$ ). We also make the formal calculations in (46) and (47) rigorous, that is we restore the higher-order terms, in particular, write the formula (47) as follows:

$$
\begin{gathered}
\left(1+\left|\frac{\varepsilon^{2}\left|\partial_{s} F(s)\right|^{2}}{(1+\varepsilon \kappa(s) F(s))^{4}}\right|\right)^{1 / 2} \frac{\partial}{\partial n^{\varepsilon}}=\frac{\partial}{\partial n}-\varepsilon \partial_{s} F(s) \frac{\partial}{\partial s}+a(\varepsilon, s) \frac{\partial}{\partial s} \\
\left|\partial_{s}^{m} a(\varepsilon, s)\right| \leq c_{m} \varepsilon, \quad m=0,1,2 \cdots
\end{gathered}
$$

Then taking the condition (48) into account yields the inequalities $\left|\widetilde{f}_{1}^{\varepsilon}(y)\right| \leq c$ and $\left|\partial_{y} \widetilde{f}_{1}^{\varepsilon}(y)\right| \leq c$, which imply

$$
\left\|\widetilde{f}_{1}^{\varepsilon} ; W_{\beta}^{1 / 2}\left(\partial \Pi^{h, \varepsilon F}\right)\right\| \leq c,
$$


in view of (56).

An argument in the next section shows that the operator $\mathcal{A}_{\beta}^{h, \varepsilon f}$ remains an isomorphism for small $\varepsilon$ and furthermore the norm of its inverse stays uniformly bounded in $\varepsilon \in\left[0, \varepsilon_{0}\right]$. The estimates (66) and (67) with $J \geq 2$ imply the inequality

$$
\left\|\widetilde{\varphi} ; \mathcal{W}_{\beta}^{2}\left(\Pi^{h, \varepsilon F}\right)\right\| \leq c .
$$

Since the norm (62) involves the coefficients $a_{ \pm}$of the expansion (61), we easily deduce from (68) and (13), (24) that

$$
|\tilde{\mathbf{t}}| \leq c \text { and }|\tilde{\mathbf{r}}| \leq c .
$$

The estimates (68) and (69) for the remainders in (45) make the formal asymptotic analysis in Sections 2 and 3 rigorous.

\subsection{Smoothness of the scattering coefficients.}

In this section we complete the proof by performing a coordinate change which turns $\Pi^{h, \varepsilon F}$ into the reference channel $\Pi^{h}$ and by treating the operators of the preceding section in $\Pi^{h}$ with a perturbation argument (cf. [15], ch. VII, §6.5). First, we make the change of curvilinear coordinates ( $F$ is as in (51))

$$
(s, n) \mapsto(s(\varepsilon, \tau), n(\varepsilon, \tau))=(s, n-\varepsilon F(s))
$$

in the tubular neighbourhood $\mathcal{V}$ of $\Gamma_{L}$, and this transforms $\Gamma_{L}^{\varepsilon}$ into $\Gamma_{L}$. In Cartesian coordinates we write the change as $(y, z) \mapsto(y(\varepsilon, \tau), z(\varepsilon, \tau))$. The domain $\Pi^{h, \varepsilon F}$ is then mapped onto the reference channel $\Pi^{h}$ by the global coordinate change

$$
\begin{aligned}
& (y, z) \mapsto(Y(\varepsilon, \tau), Z(\varepsilon, \tau)) \\
=\quad & \mathcal{X}(y, z)(y(\varepsilon, \tau), z(\varepsilon, \tau))+(1-\mathcal{X}(y, z))(y, z),
\end{aligned}
$$

where $\mathcal{X} \in C_{c}^{\infty}(\mathcal{V})$ is a cut-off function such that $\operatorname{supp} F \subset\left\{(y, z) \in \Gamma_{L} ; \mathcal{X}(y, z)=\right.$ $1\}$. The transform (70) is nonsingular and moreover "almost identical", if $\varepsilon$ and $\tau$ are small, or, belong to the cylinder (35). This means that

$$
\left|\nabla^{p}(y-Y(\varepsilon, \tau))\right|+\left|\nabla^{p}(z-Z(\varepsilon, \tau))\right| \leq c_{p}(|\varepsilon|+|\tau|), p=0,1, \ldots,
$$

and moreover that $Y(\varepsilon, \tau)$ and $Z(\varepsilon, \tau)$ depend smoothly on the parameters.

Near the free surface the Cartesian coordinates are not perturbed by the change (70). In the new coordinates the Helmholtz operator and the normal derivative differ from $-\partial_{Y}^{2}-\partial_{Z}^{2}+k^{2}$ and $\partial_{n(Y, Z)}$ only by small terms, see (70) again. The key point of our argument is that these terms have compact supports in $\mathcal{V} \cap \Pi^{h}$ and depend smoothly on $(\varepsilon, \tau) \in \mathcal{Q}$; in the framework of Section 2 the dependence is even analytic, since we had $s=y+L$ and $n=-z-d$ there. Due to compact supports, the coordinate change turns the operator $\mathcal{A}_{\beta}^{h, \varepsilon f}$ into 
$\mathcal{A}_{\beta}^{h}+\mathcal{T}_{\beta}^{h, \varepsilon F}(\tau)$, where only the small continuous addendum $\mathcal{T}_{\beta}^{h, \varepsilon F}$ depends on both $\varepsilon$ and $\tau$.

We are now in a position to apply a classical result of operator theory. Keeping in mind that $\mathcal{A}_{\beta}^{h}$ is invertible, see (63), the inverse of the operator $\mathcal{A}_{\beta}^{h}+\mathcal{T}_{\beta}^{h, \varepsilon F}$ can be presented as

$$
\left(\mathcal{A}_{\beta}^{h}+\mathcal{T}_{\beta}^{h, \varepsilon F}\right)^{-1}=\left(I+\left(\mathcal{A}_{\beta}^{h}\right)^{-1} \mathcal{T}_{\beta}^{h, \varepsilon F}\right)^{-1}\left(\mathcal{A}_{\beta}^{h}\right)^{-1},
$$

where we write the Neumann series

$$
\left(I+\left(\mathcal{A}_{\beta}^{h}\right)^{-1} \mathcal{T}_{\beta}^{h, \varepsilon F}\right)^{-1}=\sum_{n=0}^{\infty}\left(\left(\mathcal{A}_{\beta}^{h}\right)^{-1} \mathcal{T}_{\beta}^{h, \varepsilon F}\right)^{n}
$$

and $I$ denotes the identity operator $\mathcal{W}_{\beta}^{2}\left(\Pi^{h}\right) \rightarrow \mathcal{W}_{\beta}^{2}\left(\Pi^{h}\right)$. The series (73) converges in the operator norm, since the operator norm of $\left(\mathcal{A}_{\beta}^{h}\right)^{-1} \mathcal{T}_{\beta}^{h, \varepsilon F}$ : $\mathcal{W}_{\beta}^{2}\left(\Pi^{h}\right) \rightarrow \mathcal{W}_{\beta}^{2}\left(\Pi^{h}\right)$ is small. In addition to the fact that $\mathcal{A}_{\beta}^{h, \varepsilon f}$ becomes an isomorphism, we now see from (72)-(73) that the norm of its inverse is bounded uniformly in $(\varepsilon, \tau) \in \mathcal{Q}$. Moreover, the problem (57), (61) for the difference

$$
\widehat{\varphi}=\varphi-\chi_{-} w_{+} \in \mathcal{W}_{\beta}^{2}\left(\Pi^{h, \varepsilon F}\right)
$$

can be interpreted as the abstract equation

$$
\begin{aligned}
& \left(\mathcal{A}_{\beta}^{h}+\mathcal{T}_{\beta}^{h, \varepsilon F}\right) \widehat{\varphi}=\left(f, f_{0}, f_{1}\right) \quad \in W_{\beta}^{0}\left(\Pi^{h}\right) \times W_{\beta}^{1 / 2}\left(\partial \Pi^{h}\right) \times W_{\beta}^{1 / 2}\left(\partial \Pi^{h}\right),
\end{aligned}
$$

where $f_{0}=f_{1}=0$ and

$$
f(y, z)=\left(\partial_{y}^{2} \chi_{-}(y)+2 \partial_{y} \chi_{-}(y) \partial_{y}\right) w_{+}(y, z),
$$

cf. (59). Now (76) is independent of $(\varepsilon, \tau)$ and has support in the set $\{(y, z) ;-2 L \leq$ $y \leq-L, 0 \geq z \geq-d\}$ with $(y, z)=(Y, Z)$. We can thus deduce that the solution of $(75)$ in $\mathcal{W}_{\beta}^{2}\left(\Pi^{h}\right)$ depends smoothly on $(\varepsilon, \tau) \in \mathcal{Q}$ and even analytically in the case of the perturbation (18) of Section 2, because the dependence of the solution operator on $(\varepsilon, \tau)$ is smooth or analytic, due to the representation (72)-(73).

This solution is nothing but the function (74) rewritten in the coordinates $(Y(\varepsilon, \tau), Z(\varepsilon, \tau))$. Since the change (70) is the identity outside a compact set, the coefficients $\mathbf{r}$ and $\mathbf{t}$ in the norm $\left\|\widehat{\varphi} ; \mathcal{W}_{\beta}^{2}\left(\Pi^{h}\right)\right\|$, see (62), are also smooth and analytic as required in Sections 3 and 2, respectively. This completes the proofs of the existence of invisible perturbations.

\section{Extensions.}

\subsection{Set of prescribed frequencies.}

We can aim to apply a similar technique to find a perturbation of the bottom which is invisible at several frequencies. In fact, it is not possible to ensure a 
perfect transmission at different frequencies. Again, this difficulty is of the same nature as that which prevents to handle the incidence $\pi / 4$. However, what we can do is to find a perturbation of the bottom which produces no reflexions at several frequencies. We can even impose a perfect transmission at one given frequency.

More precisely, let us consider $N$ given frequencies

$$
0<\omega_{1}<\omega_{2}<\ldots<\omega_{N}
$$

Our objective is to find a profile $h=\varepsilon H$ such that

$$
\Im m\left(\mathbf{t}\left(\omega_{1}\right)\right)=0, \Im m\left(\mathbf{r}\left(\omega_{p}\right)\right)=0, \Re e\left(\mathbf{r}\left(\omega_{p}\right)\right)=0, p=1, \cdots N .
$$

Searching for a profile function in the form (18) with

$$
H(y)=H_{0}(y)+\tau_{1} H_{1}(y)+\sum_{q=1}^{N} \sum_{j=2}^{3} \tau_{q, j} H_{q, j}(y)
$$

we impose $2 N+1$ orthogonality conditions on $H_{0}$, namely

$$
\int_{-L}^{L} H_{0}(y) d y=\int_{-L}^{L} \cos \left(2 \ell_{p} y\right) H_{0}(y) d y=\int_{-L}^{L} \sin \left(2 \ell_{p} y\right) H_{0}(y) d y=0, p=1, \ldots, N
$$

where $\ell_{p}$ is determined by $\omega_{p}$ through (7) and (8). Moreover, since the constant function and the trigonometric functions $\cos \left(2 \ell_{p} y\right)$ and $\sin \left(2 \ell_{p} y\right)$ are linearly independent, we can also require the conditions

$$
\begin{gathered}
\int_{-L}^{L} H_{1}(y) d y=1, \int_{-L}^{L} \cos \left(2 \ell_{p} y\right) H_{1}(y) d y=\int_{-L}^{L} \sin \left(2 \ell_{p} y\right) H_{1}(y) d y=0, \\
\int_{-L}^{L} H_{q, j}(y) d y=0, \int_{-L}^{L} \cos \left(2 \ell_{p} y\right) H_{q, j}(y) d y=\delta_{p, q} \delta_{2, j}, \\
\int_{-L}^{L} \sin \left(2 \ell_{p} y\right) H_{q, j}(y) d y=\delta_{p, q} \delta_{3, j}, p, q=1, \ldots, N, j=2,3 .
\end{gathered}
$$

Repeating the asymptotic analysis of Section 2.1 we derive for them the formulas (27) with $\ell$ and $\gamma$ replaced by $\ell_{p}$ and $\gamma_{p}$. We also assume that $k \neq \ell_{p}$ for all $p$, which means that the angle of incidence is different from $\pi / 4$, for all frequencies. Then we can again convert the conditions

$$
\Im m\left(\mathbf{t}\left(\omega_{1} ; \tau\right)\right)=0, \Im m\left(\mathbf{r}\left(\omega_{p} ; \tau\right)\right)=0, \Re e\left(\mathbf{r}\left(\omega_{p} ; \tau\right)\right)=0
$$

for $p=1, \ldots, N$, into the equation (40) such that the operator $T^{\varepsilon}$ is still a contraction in a small ball. Thus the solution $\tau=\left(\tau_{1}, \tau_{1,2}, \tau_{1,3}, \ldots, \tau_{N, 2}, \tau_{N, 3}\right) \in$ 
$\mathbb{R}^{1+2 N}$ exists and we have detected a bottom profile which causes no reflection for the waves $w_{+}\left(\omega_{1} ; x, y\right), \ldots, w_{+}\left(\omega_{N} ; x, y\right)$ of $(12)$. We emphasize that the condition $\mathbf{t}=1$ of the intact passing wave has been achieved for one predetermined frequency $\omega_{1}$ only, but for $\omega_{p} \neq \omega_{1}$, a phase shift can occur.

Using the same approach one may consider at one given frequency $\omega$ waves with different angles of incidence corresponding to different values of the $x$ wavenumber $k<\gamma: k_{1}, \ldots, k_{M}$.

Finally, one can consider at the same time different frequencies $\omega_{1}, \ldots, \omega_{N}$ and different $x$-wavenumbers $k_{1}, \ldots, k_{M}$. In this situation, an additional condition has to be verified, to ensure the linear independence of the trigonometric functions. Frequencies and wavenumbers must be chosen such that all values $\ell\left(\omega_{p}, k_{q}\right)$ are distinct.

\subsection{Submerged fixed and freely floating bodies.}

The general method [9, Ch. 2,5,9] provides rather explicit asymptotic formulas for the velocity potential, when one or several small diameter bodies are immersed into the straight channel $\Pi$. Introducing free parameters, which for example are related to the disposition of the bodies, may lead to the same formal inferences as in Section 2. However, similarly to the case of piecewise smooth bottom profiles, a gap appears in the justification scheme because the rectification trick of $[15, \S 7.6]$ does not work any more. This is why the existence of submerged fixed or freely floating bodies, which cannot be observed by surface waves at given frequencies, remains an open problem.

\subsection{A perturbation localized in all directions.}

The reduction of the originally three dimensional water-wave problem to the two dimensional problem (9)-(11) becomes possible only when the perturbation has a strictly cylindrical shape. It seems that the perturbation technique developed here allows to deal with the full problem in the domain

$$
\Xi^{\varepsilon H}=\left\{(x, y, z):(x, y) \in \mathbb{R}^{2}, 0>z>-d-\varepsilon H(x, y)\right\},
$$

where $H$ is a compactly supported smooth function and $\varepsilon$ is a small positive

parameter. Namely, fixing a finite number of frequencies and several emitters and receivers, to find the profile $H$ such that the corresponding bottom perturbation cannot be observed by these particular surface waves and from the fixed directions. This has been proposed in the acoustic case in [16].

Acknowledgements. The second named author was partially supported by RFFI, grant 15-01-02175 and by the Academy of Finland grant no. 139545. The third named author was partially supported by the Academy of Finland project "Functional analysis and applications". 


\section{References}

[1] Schurig, D., Mock, J. J., Justice, B. J., Cummer, S. A., Pendry, J. B., Starr, A. F. and Smith, D. R., Metamaterial Electromagnetic Cloak at Microwave Frequencies, Science, 314 (2006) 977-980.

[2] Farhat, M., Enoch, S., Guenneau, S., Movchan, A. B. Broadband cylindrical cloak for linear surface waves in a fluid. Physical review letters, 101 (2008) 134501.

[3] Berraquero, C. P., Maurel, A., Petitjeans, P. and Pagneux, V. Experimental realization of a water-wave metamaterial shifter. Physical review E 88 (2013)051002(R).

[4] Bonnet-BenDhia, A.-S., Nazarov, S.A., Obstacles in acoustic waveguides becoming "invisible" at given frequencies, Acoustic Journal 59,6 (2012), 685-692. (English transl. Acoustical Physics 59 (2012) 633-639.)

[5] Porter, R., Newman, J.N., Cloaking of a vertical cylinder in waves using variable bathymetry. Journal of Fluid Mechanics 750 (2014) 124- 143.

[6] Evans, D. V., McIver, M., Porter, R., Transparency of structures in water waves, communication to IWWWFB2014 (2014).

[7] Nazarov S.A., Asymptotic expansions of eigenvalues in the continuous spectrum of a regularly perturbed quantum waveguide, Theoretical and mathematical physics 167,2 (2011), 239-262. (English transl. Theoretical and mathematical physics 167,2 (2011), 606-627.)

[8] Nazarov S.A., Enforced stability of a simple eigenvalue in the continuous spectrum of a waveguide, Funkt. Anal. i Prilozhen 47(3) (2013), 37-53.

[9] Maz'ya, V. G., Nazarov, S.A., Plamenevskii, B.A., Asymptotische Theorie elliptischer Randwertaufgaben in singulär gestörten Gebieten, vol. I, Akademie-Verlag, 1991. (English transl. Asymptotic theory of elliptic boundary value problems in singularly perturbed domains I, Birkhäuser Verlag, 2000.)

[10] Kuznetsov, N., Maz'ya, V., Vainberg, B., Linear water waves: a mathematical approach, Cambridge University Press, 2002.

[11] Leis, R., Initial boundary value problems of mathematical physics, Stuttgart, B.G. Teubner, 1986.

[12] Kondratiev, V.A., Boundary value problems for elliptic problems in domains with conical or corner points, Trudy Moskov. Matem. Obshch. 16 (1967), 209-292. (English transl. Trans. Moscow Math. Soc. 16 (1967), 227-313.) 
[13] Nazarov S.A., Properties of spectra of boundary value problems in cylindrical and quasicylindrical domains, Sobolev Spaces in Mathematics, vol. II (Maz'ya V., Ed.), Springer International Mathematical Series , 9 (2008), 261-309.

[14] Nazarov, S.A, Plamenevskii, B.A, Elliptic problems in domains with piecewise smooth boundaries, Walter be Gruyter (1994).

[15] Kato, T., Perturbation theory of linear operators, 2nd edition, Springer Verlag, 1995.

[16] Bonnet-Ben Dhia, A.-S. , Chesnel, L. and Nazarov, S.A.. Transmission eigenvalues and far field invisibility for a finite number of incident/scattering directions, to appear in Inverse Problems. 